\title{
Cost-effectiveness analysis of long-course oxaliplatin and bolus of fluorouracil based preoperative chemoradiotherapy vs. 5x5Gy radiation plus FOLFOX4 for locally advanced resectable rectal cancer
}

Shichao Wang ${ }^{1 \dagger}$, Feng Wen ${ }^{1,2,3 \dagger}$, Pengfei Zhang ${ }^{2,3}$, Xin Wang ${ }^{1,2^{*}}$ and Qiu Li $i^{2,3,4^{*}}$

\begin{abstract}
Purpose: To evaluate the cost-effectiveness of preoperative short-course radiotherapy (SCRT, $5 \times 5$ Gy) plus FOLFOX4 versus long-course oxaliplatin and bolus of fluorouracil based preoperative long-course chemoradiotherapy (LCCRT, $50.4 \mathrm{~Gy}$ in 28 fractions) in the management of cT4 or advanced CT3 rectal cancer (RC), both of which have been reported to achieve similar clinical effect in the NCT00833131 trial.
\end{abstract}

Materials and methods: A Markov decision-analytic model compared SCRT plus chemotherapy and LCCRT, by simulating three health states (disease-free survival (DFS), progressive disease (PD) and death). The primary outcomes were quality-adjusted life months (QALMs), costs, and incremental cost-effectiveness ratios (ICERs). Transition probabilities were based on the NCT00833131 trial. The costs were calculated from a Chinese payers' perspective. Strategies were evaluated with a willingness-to-pay (WTP) threshold of $\$ 2370.47$ (3 × GDP) per QALM gained. Sensitivity analysis was performed to model uncertainty in these parameters.

Results: The overall costs for SCRT plus chemotherapy and LCCRT were $\$ 78,937$ and $\$ 38,140$ with effectiveness of 29.92 QALMs and 22.99 QALMs, respectively. SCRT plus chemotherapy increased costs and QALM by $\$ 40,797.34$ and 6.93 compared to LCCRT, resulting in an ICER of $\$ 5884.56 /$ QALM gained. In the DFS state, the whole cost for SCRT plus chemotherapy and LCCRT were $\$ 11,490.03$ and $\$ 10,794.06$ with an effectiveness of 21.70 QALMs and 19.65 QALMs, respectively. SCRT plus chemotherapy increased cost and QALM by $\$ 695.97$ and 2.05 compared to LCCRT, resulting in a ICER of $\$ 339.50 /$ QALM gained, which below the WTP. The utility associated with the DFS state was the most influential factor on the cost-effectiveness of SCRT plus chemotherapy. When the cost of PD state below \$1920, the ICER of SCRT compared with LCCRT below the WTP.

Conclusion: Compared with LCCRT, SCRT plus chemotherapy is a more cost-effective strategy for locally advanced resectable RC in the DFS state as well as in the all states when the cost of PD state below \$1920.

Keywords: Cost-effectiveness, Short-course radiotherapy, Long-course chemoradiotherapy, Rectal cancer, Hypofractional radiotherapy

\footnotetext{
* Correspondence: wangxinphy@163.com; fbqiu9@163.com

†Shichao Wang and Feng Wen contributed equally to this work.

'Department of Radiation Oncology, Cancer Center and State Key Laboratory

of Biotherapy, West China Hospital, Sichuan University, Chengdu, Sichuan

Province, People's Republic of China

${ }^{2}$ Department of Abdominal Oncology, Cancer Center and State Key

Laboratory of Biotherapy, West China Hospital, Sichuan University, 37\# of

Wainan Guoxue Lane, Chengdu, Sichuan Province 610041, People's Republic

of China

Full list of author information is available at the end of the article
}

(c) The Author(s). 2019 Open Access This article is distributed under the terms of the Creative Commons Attribution 4.0 International License (http://creativecommons.org/licenses/by/4.0/), which permits unrestricted use, distribution, and reproduction in any medium, provided you give appropriate credit to the original author(s) and the source, provide a link to the Creative Commons license, and indicate if changes were made. The Creative Commons Public Domain Dedication waiver (http://creativecommons.org/publicdomain/zero/1.0/) applies to the data made available in this article, unless otherwise stated. 


\section{Introduction}

Rectal cancer (RC) is one of the most frequent malignancies in the world and represents a major socioeconomic and health issue [1]. In China, colorectal cancer (CRC) has attracted increasing attention over recent years, taking a second and fourth position in the incidence and mortality respectively among all malignant tumors in urban populations [2]. RC accounts for about $40 \%$ in the morbidity of CRC [2].

Surgery is the basic treatment for RC, but for advanced RC, adjuvant radiotherapy with or without chemotherapy has been used widely to improve outcomes. It is well known for locally advanced disease, postoperative chemoradiotherapy (CRT) significantly improves both local control and overall survival as compared with surgery alone or surgery plus irradiation [3, 4]. There are fewer studies on the preoperative treatments. Preoperative short-course radiotherapy (SCRT) plus chemotherapy consisting of FOLFOX4 or preoperative long-course chemoradiotherapy (LCCRT) with oxaliplatin and boluses of 5- fluorouracil and leucovorin in combination with conventional surgery are recommended depending on the tumor location, infiltration depth of the tumor, and lymph node involvement, which improves local control and survival [5]. Early results showed shortterm preoperative radiotherapy decreased risk of local recurrence for irradiated patients at 2 years ( $2 \% \mathrm{vs} 8 \%, p<$ 0.001 ) without a difference in overall survival (OS) for the patients with rectal cancer who undergo a standardized total mesorectal excision [6]. After a median follow-up of 6 years, the effect of radiotherapy on local recurrence persisted ( $6 \%$ vs $11 \%, p<0.001)$, as well as the absence of a survival benefit for the patients with mobile rectal cancer undergoing surgery treated with preoperative short-term radiotherapy [7]. There is no international consensus on the use of these treatment schedules or the most appropriate patient selection for these schedules.

The phase III trial NCT00833131 comparing SCRT $(5 \times$ 5 Gy) consisting of FOLFOX4 versus LCCRT $(1.8 \times 28 \mathrm{~Gy})$ in patients with fixed cT3 or cT4 rectal cancer with oxaliplatin and boluses of 5- fluorouracil and leucovorin, revealed that preoperative LCCRT, aimed at tumor shrinkage, is used to achieve R0 resection [8]. Nevertheless, improved OS and lower acute toxicity favours the $5 \times$ 5 Gy schedule with consolidation chemotherapy, with statistically-significant improvements found in diseasefree survival (DFS) and OS with SCRT $(p=0.046)$ [8].

Several studies (e.g. CAO/ARO-094) have proved that preoperative radiotherapy has advantages in promoting the local control rates (LCR) and reducing toxicity compared with other treatment methods [9-13]. So far, no economic evaluation is available that compares preoperative SCRT with consolidation chemotherapy versus preoperative LCCRT. It is reported that SCRT with consolidation chemotherapy could be considered as an effective option for preoperative management in advanced rectal cancer, especially in countries with low health care budgets or long waiting lists for radiotherapy [8]. Given the increasing emphasis on value-based care in oncology, the aim of this study was to compare SCRT plus chemotherapy and LCCRT for the management of cT4 or advanced cT3 RC using a decision analysis approach.

\section{Materials and methods Decision model}

A Markov model was used to estimate the overall benefits, risks and costs of SCRT plus chemotherapy compared to LCCRT by simulating a cohort of patients with rectal cancer over a 10 year time horizon. Model parameters were based on the literature and clinical data. TreeAge Pro 2016 (TreeAge Inc., Williamstown, Mass) was used for the Markov model.

In this study, we defined three health states: DFS, progressive disease (PD), and death (Fig. 1). A patient was considered to be in one of these health states at any given time. The Markov model was based on a payer's perspective and ran with a 1-month cycle length.

All patients began in the DFS state after the CRT and surgery treatments, and had monthly potential transitions to PD or death. SCRT consisted with either preoperative 5 $\times 5$ Gy irradiation over 5 days with consolidation chemotherapy consisting 3 cycles of FOLFOX4. LCCRT consisted with preoperative LCCRT consisting $1.8 \times 28 \mathrm{~Gy}$ concomitantly with oxaliplatin and boluses of 5- fluorouracil and leucovorin. Surgery costs were also included in both arms of this study.

This is a retrospective study was approved by medical ethics committee of West China Hospital, Sichuan University, People's Republic of China. None of the authors directly interacted with any study participants as the analysis was model-based and relied on inputs from the published literature and medical databases.

\section{Health state transitions}

The monthly transition probabilities between the states were based on the Kaplan-Meier survival curves for DFS and OS for both strategies from the NCT00833131 trial [8]. To extrapolate the probability of survival during the observation period, Engauge Digitizer software was used to extract digitized data points from the Kaplan-Meier curves from the study. The Microsoft excel solver were used to calibrate 2-parametric Weibull survival models to the observed trial data by minimizing the sum of monthly squared deviations between the curves over a 6 year time horizon. 


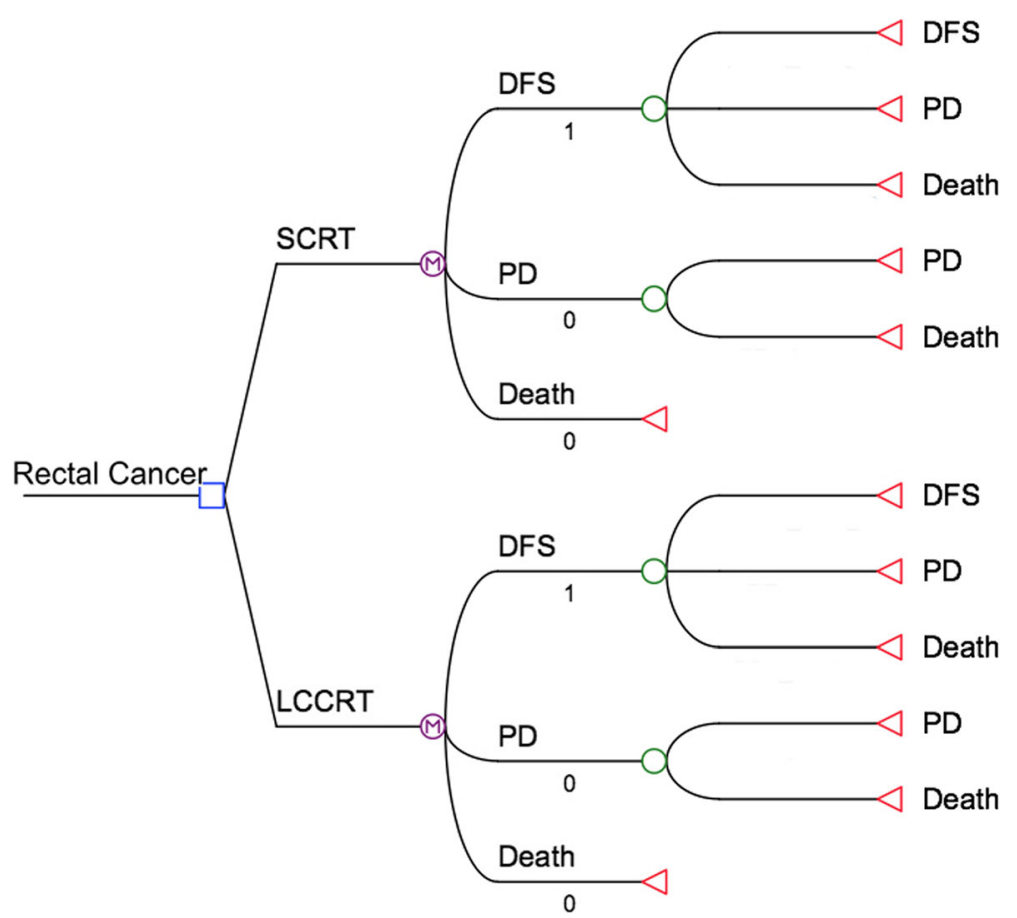

Fig. 1 Markov model for advanced rectal cancer. According to the study profile, two groups were analyzed: group SCRT, patients with locally advanced resectable rectal cancer treated with preoperative short-course irradiation $(5 \times 5 \mathrm{~Gy})$ and immediate surgery plus chemotherapy; group LCCRT, patients with locally advanced resectable rectal cancer treated with long-course preoperative CRT $(1.8 \times 28 \mathrm{~Gy})$ and delayed surgery. A Markov model comprising three health states (disease-free survival (DFS), progressive disease (PD) and death) was built

\section{Cost}

Direct medical costs were considered as part of the payer perspective, which included costs of radiotherapy, chemotherapy drugs, surveillance (physician visit, blood tests, serum carcinoembryonic antigen (CEA) level test, computed tomography (CT) and colonoscopy), medical care (cost of care for metastatic CRC), adverse events. A detailed assessment of unit costs for these resources was conducted using data from the Cancer Center of West China Hospital of Sichuan University. All costs were converted into 2018 U.S. dollars based on the medical price index of National Health and Family Planning Commission of People's Republic of China (PRC) and an exchange rate of $\$ 1=¥ 6.5$ (Jun., 2018).

The total direct cost of radiotherapy was determined by multiplying resource use with the unit costs. Indirect department costs and overheads were allocated to treatments based on the number of fractions delivered. The total cost of radiotherapy was considered in the first cycle of the Markov model. The societal costs, such as travel costs and time costs, were not included in this study.

Because of limited information about the treatments for patients who were suffered from recurrence after the surgery in the NCT00833131 trial, the costs in the PD state were based on FIRE-3, a previously-published costeffectiveness analysis from the Chinese perspective [14].

\section{Cost-effectiveness and utilities}

The utility of the DFS and the PD state were based on published research. According to Ramsey's study about colorectal cancer patients who received surgery [15], the utility of DFS was set at 0.84 . The utility of the PD state was 0.6 based on Wen's study [14]. Considering the treatment strategies of both groups were similar, the utilities for each state of both groups were assumed to be the same. The utilities were used to compute total quality-adjusted life months (QALMs) for each treatment [16]. The QALM is a combination of length of life and quality of life, with each month of life weighted by the utility that reflects the quality of life.

The incremental cost-effectiveness ratio (ICER) was calculated by dividing the difference in costs by the difference in QALMs. We considered both benefits and costs from a social perspective regardless of to whom they accrued and discounted both at a 3\% annual rate. The cost-effectiveness analysis was carried out from a Chinese payer's perspective. Model transition probabilities, costs, and utilities are included in Table 1. 
Table 1 Transition probabilities, unit costs, and utilities used in the analysis

\begin{tabular}{llll}
\hline Parameter & SCRT & LCCRT & Data source \\
\hline $\begin{array}{l}\text { Survival } \\
\text { 3 year-DFS }\end{array}$ & $53 \%$ & $52 \%$ & Bujko et al. [8] \\
$\begin{array}{l}\text { 3-year OS } \\
\text { Probabilities (monthly) }\end{array}$ & $73 \%$ & $65 \%$ & Bujko et al. [8] \\
DFS to PD & 0.0204 & 0.0244 & \\
$\begin{array}{l}\text { DFS to Death } \\
\text { PD to Death }\end{array}$ & 0.0148 & 0.0148 & \\
Costs per Cycle in US\$ & 0.0348 & 0.1020 & \\
DFS state & 444.85 & 461.34 & \\
PD state & 4920.50 & 4920.50 & Wen et al. [14] \\
Utility & & & \\
DFS state & 0.65 & 0.56 & Ramsey et al. [15] \\
PD state & 0.47 & 0.47 & Wen et al. [14] \\
Death state & 0.00 & 0.00 &
\end{tabular}

Abbreviations: SCRT Short-course radiotherapy, LCCRT Long-course chemoradiotherapy, DFS Disease-free survival, OS Overall survival, PD Progressive disease, $m$ median

\section{Sensitivity analysis}

We carried out a one-way sensitivity analysis to examine the influence of different parameters on the overall costeffectiveness of treatment. The variables in the sensitivity analysis varied at a range of $\pm 20 \%$. In addition, to account for overall uncertainty we conducted a probabilistic sensitivity analysis were all parameters are varied simultaneously in a Monte Carlo simulation of 10,000 individuals and 1000 trials. Those results were reported as the percentage of trials in which a strategy is costeffective at a series of willingness-to-pay (WTP) (or acceptability) thresholds in terms of dollars spent per QALM gained. We particularly focused on a threshold of $3 \times$ the per capita GDP of China, which comports with the WHO guidelines for cost-effectiveness analyses [17]. Per capita disposable income of Chinese patients in 2017 was $\$ 790$ per month, so the WTP threshold was $\$ 2370.47$ (3 × GDP) per QALM.

\section{Results}

\section{Patients characteristics}

The Polish Colorectal Cancer Study Group enrolled patients in an open labeled, prospective, randomized phase III trial NCT00833131. Five hundred fifteen patients from 39 Polish institutions were randomly assigned either to SCRT plus chemotherapy or LCCRT. Two hundred sixty-one allocated to $5 \times 5$ Gy plus chemotherapy, 254 allocated to LCCRT among them. Based on the study, the 3 year disease-free survival and overall survival in SCRT plus chemotherapy and LCCRT were 53\% vs. $52 \%(p=0.85)$ and $73 \%$ vs. $65 \%(p=0.046)$, respectively.

\section{Cost}

SCRT plus chemotherapy cost much more than LCCRT $(\$ 78,937$ vs. $\$ 38,140)$. Costs in the DFS state were slightly higher with SCRT plus chemotherapy than in LCCRT $(\$ 11,490$ vs. $\$ 10,794)$ because of the slightly higher disease-free survival. Because overall survival was higher with SCRT plus chemotherapy, the costs accrued in the PD state were much higher with SCRT plus chemotherapy than LCCRT $(\$ 67,447$ vs. $\$ 27,346)$. Overall, the incremental cost of SCRT plus chemotherapy over LCCRT was $\$ 40,797$. Information regarding CRT and RT analyzed in the model is illustrated in Table 2. The costs of radiotherapy were considerably cheaper compared with chemotherapy agents in general.

\section{Effectiveness}

The total effectiveness was 29.92 QALMs for SCRT plus chemotherapy, and 22.99 QALMs for LCCRT. In detail, 21.27 QALMs were accrued in the DFS state and 8.22 QALMs in the PD state for SCRT plus chemotherapy. With LCCRT, 19.65 QALMs and 3.33 QALMs were accrued in DFS and PD states, respectively.

\section{Cost-effectiveness}

Table 3 presented the results of the cost-effectiveness analysis. Incremental costs were \$ 40797 and the incremental effectiveness was 6.93 QALMs in patients treated in SCRT plus chemotherapy compared with patients who received in LCCRT, resulting in an ICER of \$5885/ QALM gained (Fig. 2a).

\section{Sensitivity analysis}

One-way and probabilistic sensitivity analyses were performed to test the robustness of the Markov model. The variables in the sensitivity analysis varied at a range of \pm $20 \%$, and the results are shown as the tornado diagram in Fig. 3. Net monetary benefit (NMB) was applied in the tornado diagram. According to the calculation formula NMB $=$ Effectiveness $\times$ WTP-Cost, NMB combines cost, effectiveness and WTP into a single measurement, and can show at what point in variable range do we have a change in the recommended strategy based on costeffectiveness (Fig. 2b) . The WTP is set at $\$ 2370.47(3 \times$ GDP) per QALM.

Results of the sensitivity analysis revealed that the utility of DFS state was the most influential factor for the robustness of the model, followed by transition probabilities in SCRT plus chemotherapy and LCCRT. On sensitivity analysis, as shown in Fig. 2a, there was a linear relationship between the feasibility rate of highconfidence differentiation between the two groups on the Chinese population, for testing the responsiveness of the model and the robustness of our results. 
Table 2 Cost for in US\$ chemotherapy, hospitalization, test and surgery per patient

\begin{tabular}{|c|c|c|}
\hline Cost item & SCRT & LCCRT \\
\hline \multicolumn{3}{|l|}{ Radiotherapy } \\
\hline Physician Consultation & 14.7 & 14.7 \\
\hline CT Simulation & $470+5.3$ & $470+5.3$ \\
\hline Physics planning & 177 & 177 \\
\hline Treatment and positioning & 3032 & 4928 \\
\hline Number of fractions delivered & 5 & 28 \\
\hline \multicolumn{3}{|l|}{ Drug (Direct) } \\
\hline Frequency & FOLFOX4 & 5-fluorouracil: $325 \mathrm{mg} / \mathrm{m}^{2} /$ day leucovorin: $25 \mathrm{mg} / \mathrm{m}^{2} /$ day \\
\hline For the DFS state/m & 39.31 & 38.16 \\
\hline For the PD state/m & 79.33 & 57.95 \\
\hline Total cost & 7064.01 & 5670.94 \\
\hline \multicolumn{3}{|l|}{ Hospitalization } \\
\hline Caregiver (nurse) & 52/day & 52/day \\
\hline \multicolumn{3}{|l|}{ Tests } \\
\hline Surgery & 2963.97 & 2645.13 \\
\hline \multicolumn{3}{|l|}{ Perioperative } \\
\hline$A E$ & 6.12 & 5.49 \\
\hline Base+Follow Up & $396.25+3357.58$ & $330.04+3357.58$ \\
\hline
\end{tabular}

Abbreviations: SCRT Short-course radiotherapy, LCCRT Long-course chemoradiotherapy, CT Computed tomography, PD Progressive disease, DFS Disease-free survival, $A E$ Adverse event

Because the cost of PD state was borrowed from our previous study, it was a important factor affecting the results of our model. It was found that when the cost of PD state below \$1920, the ICER of SCRT plus chemotherapy compared with LCCRT below the WTP (Fig. 4).

\section{Discussion}

There have been plenty of studies published on the preoperational CRT regimens for $\mathrm{RC}$ which have been widely used in clinical practice. However, the research

Table 3 Results of cost-effectiveness analysis

\begin{tabular}{lll}
\hline Parameter & SCRT & LCCRT \\
\hline Cost in US\$ & & \\
Costs for the DFS state & 14490 & 10794 \\
Costs for the PD state & 67447 & 27346 \\
Total costs in US\$ & 78937 & 38140 \\
Incremental costs & 40797 & \\
Utilities & & \\
Effectiveness (QALMs) & & 19.65 \\
Effectiveness for the DFS state & 21.70 & 3.33 \\
Effectiveness for the PD state & 8.22 & 22.99 \\
Total effectiveness & 29.92 & \\
Incremental effectiveness & 6.93 & \\
\hline
\end{tabular}

Abbreviations: SCRT Short-course radiotherapy, LCCRT Long-course chemoradiotherapy, PD Progressive disease, DFS Disease-free survival, QALM Quality-adjusted life month on economic analysis was rarely reported focusing the cost-effectiveness comparison. This is the first study to specifically investigate the cost-effectiveness between the preoperational SCRT plus chemotherapy and LCCRT for advanced RC patients.

One of the strengths of this study is that we included that preoperative SCRT plus chemotherapy consisting 3 cycles of FOLFOX4 may be a more cost-effective for locally advanced resectable RC, especially for the patients in the DFS state. The SCRT plus chemotherapy had more total cost than LCCRT with 5-fluorouracil and leucovorin, which maybe resulted from the long survival in PD state after the treatment of DFS state and the high cost of targeted therapies in PD state. But for the patients in DFS, the whole cost for SCRT plus chemotherapy and LCCRT were $\$ 11,490$ and $\$ 10,794$ with an effectiveness of 21.70 QALMs and 19.65 QALMs, respectively. In other words, SCRT plus chemotherapy increased cost and QALM by $\$ 695.97$ and 2.05 relative to LCCRT, resulting in a ICER of $\$ 339.50 /$ QALM gained, which below the WTP. Besides, our result was markedly consistent on deterministic and probabilistic sensitivity analyses, strengthening the argument that this novel technology is actually cost-effectiveness. The challenges facing healthcare worldwide is the incremental costeffectiveness and the threshold for using or rejecting specific drugs. Based on the trial, SCRT with consolidation chemotherapy can be considered as an effective 


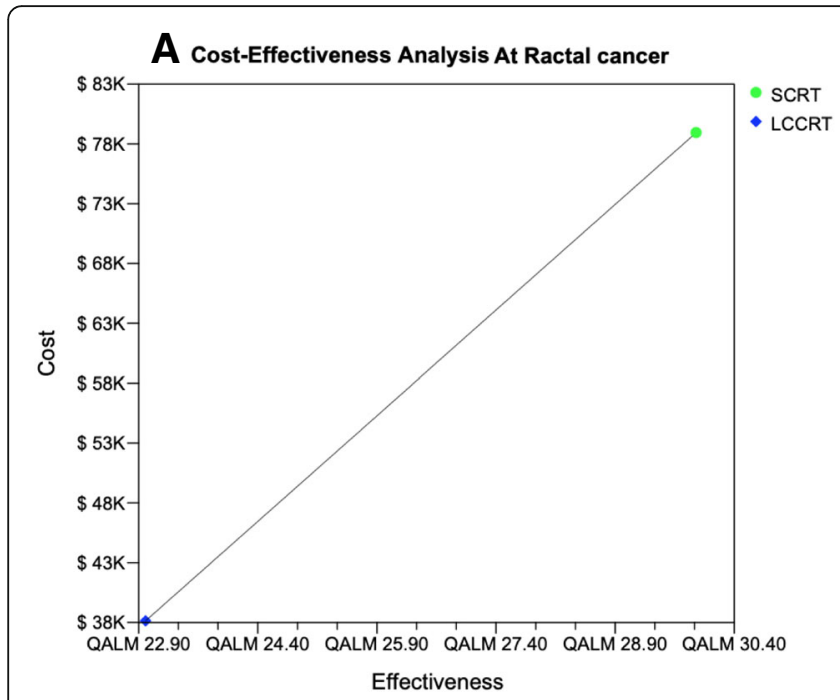

B

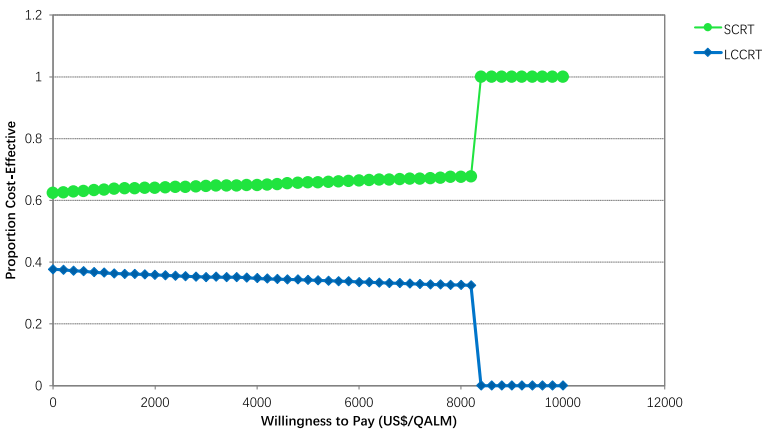

Fig. 2 a: Cost-effectiveness pictured with two groups. Two groups were analyzed: group SCRT and group LCCRT patients with advanced resectable rectal cancer. b: Cost-effectiveness acceptability curves for SCRT and LCCRT strategies. Each curve shows the probability that the ICER for each treatment falls within a given willingness-to-pay threshold. QALM, Quality-adjusted life year; US\$, United States dollars

option for preoperative management in advanced RC, especially in countries with low health care budgets or long waiting lists for radiotherapy. Similar results were concluded by Mandy Van Den et al that short-term preoperative radiotherapy in patients with rectal cancer undergoing total mesorectal excision (TME) is both effective and cost-effective based on the trial conducted by the Dutch Colorectal Cancer Group [18]. And Dahlberg $M$ et al found $5 \times 5$ Gy preoperative radiotherapy was in the range of other well-accepted medical interventions with reduced local recurrence rates and improved overall survival in a Swedish Rectal Cancer Trial [19].

On sensitivity analysis, the utility of DFS state was the most influential factor for the robustness of the model, followed by transition probabilities in SCRT plus chemotherapy and LCCRT. Especially, the costs in perioperative period had the greater impact on the results than the radiotherapy only. Furthermore, a key factor distinguishing between the two groups was the time arrangement for radiotherapy and chemotherapy, except for the surgery. Since SCRT plus chemotherapy costs much

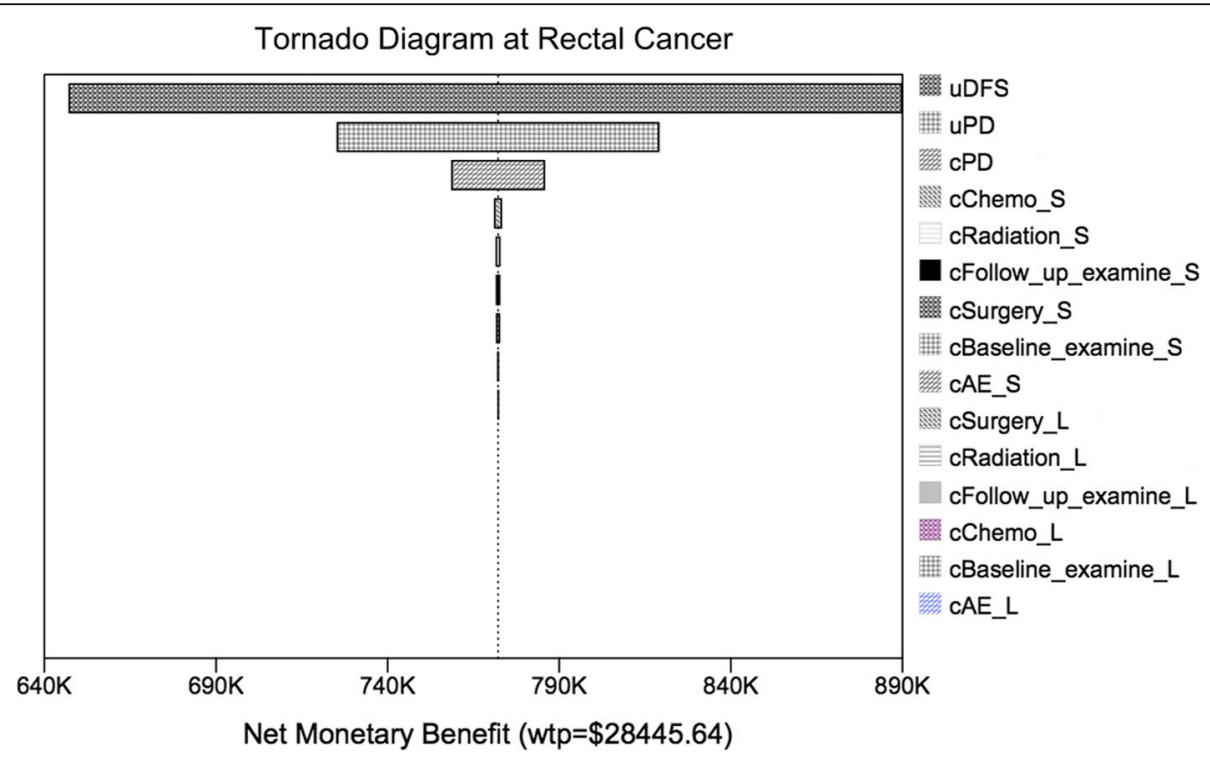

Fig. 3 Tornado diagram of one-way sensitivity analysis. Tornado diagram summarized the results of one-way sensitivity analysis to identify model variables associated with the two strategies in the treatment of advanced gastric cancer 


\section{Sensitivity Analysis on cost of PD state}

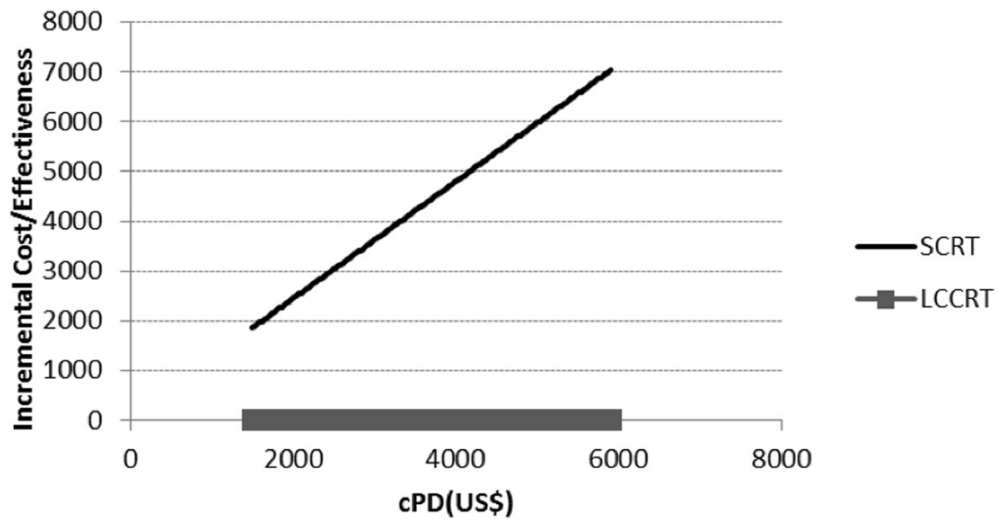

Fig. 4 Sensitivity analysis on cost of PD state of two groups

more than LCCRT ( $\$ 78,937$ vs. $\$ 38,140)$, cost for DFS state was higher in SCRT plus chemotherapy than in LCCRT, while cost for PD state was distinctly higher in SCRT plus chemotherapy than in LCCRT. There more chemotherapy drugs were given by infusion in LCCRT, which affected the stay-days in hospital. The costs of supportive drugs also showed a profound impact on the analysis model, which were related to patients' length of stay. The longer the hospital stay, the more supportive drugs were administrated. It was suggested that the hospitals losses could be reduced by shortening the length of patients' hospitalization. Dramatically, QALMs in the PD state for SCRT plus chemotherapy was distinctly higher than the data in LCCRT (8.22 vs. 3.33). There may be a balance between the cost and effectiveness after surgery. The results were consistent with Nishimura et al. [20], which suggested that the hospitals losses could be reduced by shortening the length of patients' hospitalization, furthermore, increasing the dose per fraction is a good choice for the patient with RC.

Several limitations may not been ignored in this study. Principally, this study was based on a published clinical trial, rather than a randomized prospective research. It reflects the actual resource use of the subjects' care but, of course, is subject to bias. Furthermore, the information about the PD state in the NCT00833131 trial was limited, the costs in this setting were based on the costeffectiveness analysis from Chinese FIRE-3 study, which has been analyzed by our group previously. Data from many different sources could be reasonably combined into a single model to yield useful results, but this may have determined multiple biases in the analysis. For example, the adverse events maybe changed because of the different nationality in NCT00833131 trial and FIRE3(Polish vs. Germany and Austria), leading to little cost discrepancy in the PD state. It's worth noting that treatments to PD state is palliative care for advanced rectal cancer, so when the patients in the NCT00833131 trial translated to PD state, the strategies could be the same as patients in FIRE-3. At the same time, costeffectiveness analysis for FIRE-3 study was from Chinese perspective, which was the same in the current study, so there was no nationality difference. Undeniably, although we assessed the economic costs in this study, it was reminded that some other factors biasing the analysis result would influence the choice of fit treatment protocol for the patients with $\mathrm{RC}$, containing the time away from home, lost productivity costs, education, religion. Meanwhile, as Dahlberg et al concluded that the long-term side effects of radiotherapy and chemotherapy before surgery should be of future prime concern [19]. Based on these limitations, we still believe that our study will encourage decision makers to take a more comprehensive view of treatment- related costs.

\section{Conclusion}

Compared to LCCRT, SCRT plus chemotherapy was more cost-effective for locally advanced resectable RC, despite the higher cost with SCRT plus chemotherapy has been shown in this study. Our analysis sharply suggested that it should be preferred to hypofractional radiotherapy in a cost-conscious environment for the patients in the DFS state as well as in the all states when the cost of PD state below $\$ 1920$.

\section{Abbreviations}

CEA: Carcinoembryonic antigen; CRC: Colorectal cancer; CRT: Chemoradiotherapy; CT: Computer tomography; DFS: Disease-free survival; GDP: Gross domestic product; ICER: Incremental cost-effectiveness ratio; LCCRT: Long-course chemoradiotherapy; NMB: Net monetary benefit; OS: Overall survival; PD: Progressive disease; QALM: Quality-adjusted life month; RC: Rectal cancer; SCRT: Short-course radiotherapy; TME: Total mesorectal excision; WTP: Willingness-to-pay

\section{Acknowledgements}

We take this opportunity to show our sincere gratitude to Dr. Xue, who has given this study so much useful advices on writing, and tried his best to 
improve this paper. We would like to thank Prof. David, from University of Michigan School of Public Health, for providing language editing. With his help, we have revised the entire paper, but most importantly, the methods, results, and discussion, to make the English more readable.

\section{Authors' contributions}

All authors contributed to the manuscript. All authors approve the final version.

\section{Funding}

Supported by grants from Science and Technology Department of Sichuan Province of China (2019YFS0341).

\section{Availability of data and materials}

The dataset generated and analyzed during the current study are available from the corresponding author on reasonable request.

\section{Ethics approval and consent to participate}

Not applicable.

\section{Consent for publication}

Not applicable.

\section{Competing interests}

The authors declare that they have no competing interests.

\section{Author details}

'Department of Radiation Oncology, Cancer Center and State Key Laboratory of Biotherapy, West China Hospital, Sichuan University, Chengdu, Sichuan Province, People's Republic of China. ${ }^{2}$ Department of Abdominal Oncology, Cancer Center and State Key Laboratory of Biotherapy, West China Hospital, Sichuan University, 37\# of Wainan Guoxue Lane, Chengdu, Sichuan Province 610041, People's Republic of China. ${ }^{3}$ West China Biostatistics and Cost-Benefit Analysis Center, Sichuan University, Chengdu, Sichuan Province 610041, People's Republic of China. ${ }^{4}$ Department of Medical Oncology, Cancer Center, State Key Laboratory of Biotherapy, West China Hospital, Sichuan University, 37\# of Wainan Guoxue Lane, Chengdu, Sichuan Province 610041, People's Republic of China.

Received: 20 February 2019 Accepted: 7 June 2019

Published online: 24 June 2019

\section{References}

1. Jemal A, Siegel R, Ward E, Hao Y, Xu J, Thun M. Cancer statistics, 2009. CA Cancer J Clin. 2009:59(4):225-49.

2. Zhao P, Chen WQ. Chinese Cancer registry annual report 2010 China. Beijing: Military Medical Science Press; 2011. p. 32-8.

3. Gastrointestinal Tumor Study Group. Prolongation of the disease-free interval in surgically treated rectal carcinoma. N Engl J Med. 1985;312(23): 1465-72.

4. Krook JE, Moertel CG, Gunderson LL, Wieand HS, Collins RT, Beart RW, et al. Effective surgical adjuvant therapy for high-risk rectal carcinoma. N Engl J Med. 1991;324(11):709-15.

5. Swedish Rectal Cancer Trial, Cedermark DM, Glimelius B, Påhlman L, Rutqvist $L E$, et al. Improved survival with preoperative radiotherapy in resectable rectal cancer. N Engl J Med. 1997;336(14):980-7.

6. Kapiteijn E, Marijnen CA, Nagtegaal ID, Putter H, Steup WH, Wiggers T, et al. Preoperative radiotherapy combined with total mesorectal excision for resectable rectal cancer. N Engl J Med. 2001;345(9):638-46.

7. Peeters KC, Marijnen CA, Nagtegaal ID, Kranenbarg EK, Putter H, Wiggers T, et al. The TME trial after a median follow-up of 6 years: increased local control but no survival benefit in irradiated patients with resectable rectal carcinoma. Ann Surg. 2007;246(5):693-701.

8. Bujko K, Wyrwicz L, Rutkowski A, Malinowska M, Pietrzak L, Kryński J, et al. Long-course oxaliplatin-based preoperative chemoradiation versus $5 \times 5$ Gy and consolidation chemotherapy for CT4 or fixed cT3 rectal cancer: results of a randomized phase III study. Ann Oncol. 2016;27(5):834-42.

9. Sauer R, Becker H, Hohenberger W, Rödel C, Wittekind C, Fietkau R, et al. Preoperative versus postoperative chemoradiotherapy for rectal cancer. $\mathrm{N}$ Engl J Med. 2004;351(17):1731-40.
10. Bosset JF, Collette L, Calais G, Mineur L, Maingon P, Radosevic-Jelic L, et al. Chemotherapy with preoperative radiotherapy in rectal cancer. N Engl J Med. 2006;355(11):1114-23.

11. Roh MS, Colangelo LH, O'Connell MJ, Yothers G, Deutsch M, Allegra CJ, et al. Preoperative multimodality therapy improves disease-free survival in patients with carcinoma of the rectum: NSABP R-03. J Clin Oncol. 2009; 27(31):5124-30.

12. Park JH, Yoon SM, Yu CS, Kim JH, Kim TW, Kim JC. Randomized phase 3 trial comparing preoperative and postoperative chemoradiotherapy with capecitabine for locally advanced rectal cancer. Cancer. 2011;117(16):3703-12.

13. Sauer R, Liersch T, Merkel S, Fietkau R, Hohenberger W, Hess C, et al. Preoperative versus postoperative chemoradiotherapy for locally advanced rectal cancer: results of the German CAO/ARO/AIO-94 randomized phase III trial after a median follow-up of 11 years. J Clin Oncol. 2012;30(16):1926-33.

14. Wen F, Yang Y, Zhang P, Zhang J, Zhou J, Tang R, et al. Cost-effectiveness of RAS screening before monoclonal antibodies therapy in metastatic colorectal cancer based on FIRE3 study. Cancer Biol Ther. 2015:16(11):1577-84.

15. Ramsey SD, Andersen MR, Etzioni R, Moinpour C, Peacock S, Potosky A, et al. Quality of life in survivors of colorectal carcinoma. Cancer. 2000; 88(6):1294-303.

16. Gold M, Siegel J, Russell L, et al. Cost-effectiveness in health and medicine. New York: Oxgord University Press; 1996. p. 425.

17. Murray CJ, Evans DB, Acharya A, Baltussen RM. Development of WHO guidelines on generalized cost-effectiveness analysis. Health Econ. 2000;9(3): 235-51.

18. Van Den Brink M, Van Den Hout WB, Stiggelbout AM, Klein Kranenbarg E, Marijnen CA, Van De Velde CJ, et al. Cost-utility analysis of preoperative radiotherapy in patients with rectal cancer undergoing total mesorectal excision: a study of the Dutch colorectal Cancer group. J Clin Oncol. 2004; 22(2):244-53.

19. Dahlberg M, Stenborg A, PåhIman L, Glimelius B, Swedish Rectal Cancer Trial. Cost-effectiveness of preoperative radiotherapy in rectal cancer: results from the Swedish rectal Cancer Trial. Int J Radiat Oncol Biol Phys. 2002;54(3): 654-60.

20. Nishimura Y, Mitsutake N, Nakanishi S, Konishi T. Cost analysis on stomach cancer treatment in Japanese hospitals. Gan To Kagaku Ryoho. 2000;27(9): $1360-8$

\section{Publisher's Note}

Springer Nature remains neutral with regard to jurisdictional claims in published maps and institutional affiliations.

Ready to submit your research? Choose BMC and benefit from:

- fast, convenient online submission

- thorough peer review by experienced researchers in your field

- rapid publication on acceptance

- support for research data, including large and complex data types

- gold Open Access which fosters wider collaboration and increased citations

- maximum visibility for your research: over $100 \mathrm{M}$ website views per year

At BMC, research is always in progress.

Learn more biomedcentral.com/submissions 